Section II

\title{
Histoire sociolinguistique de la Polynésie française
}

La situation sociolinguistique de la Polynésie française est complexe et hétérogène. L'unité linguistique de l'archipel n'a sans doute jamais existé : le peuplement se serait fait très vraisemblablement par vagues d'immigration successives de populations parlant des dialectes apparentés - puisque tous polynésiens - mais tous déjà différenciés dès l'époque de leur dispersion.

Aux divisions linguistiques initiales des langues polynésiennes dans chaque région, se sont ajoutés - surtout dans les archipels des Tuamotu et des Australes - l'adstrat tahitien, puis plus récemment au cours des cinquante dernières années, le superstrat français. (Une langue X est un ADSTRAT par rapport à une langue $\mathrm{Y}$, si elle est parlée dans une région voisine, et l'influence d'une manière ou d'une autre. Quant au SUPERSTRAT, il désigne toute langue qui s'introduit très largement dans l'aire d'une autre langue, mais sans s'y substituer, et qui peut disparaître finalement en laissant quelques traces.)
Cette évolution, qui se poursuit aujourd'hui, a créé une "triglossie" - cohabitation de trois variétés linguistiques sur un même territoire, y compris pour un même locuteur. Au sommet, le FRANÇAIS, langue officielle partout enseignée et à tous les niveaux; puis le TAHITIEN, langue de prestige, plus ou moins en conflit avec le français dans les domaines administratifs et politiques, et parfois ressentie comme envahissante dans les autres archipels (Marquises, Tuamotu) ; enfin, les divers VERNACULAIRES polynésiens.

En Polynésie française, pays plurilingue, la situation sociolinguistique est instable et dynamique. Elle révèle un état d'équilibre ponctuel entre différentes langues (langue officielle, lingua franca régionale, vernaculaires locaux). Ce sont essentiellement des forces extra-linguistiques - économiques, politiques, religieuses - qui, en transformant la société, conduisent à des hiérarchisations nouvelles entre langues, donnant lieu d'une part à une TAHITIANISATION du pays, et d'autre part à sa FRANCISATION.

\section{Les mécanismes historiques de la tahitianisation}

On peut appeler "tahitianisation" le processus linguistique multiforme par lequel certains dialectes locaux de l'archipel remplacent progressivement une partie de leur vocabulaire, ou de leur phonologie, par les éléments du dialecte dominant, celui de l'île de Tahiti. Ce phénomène, fort ancien, remonte au tout début du XIX ${ }^{e}$ siècle, alors que débutait l'expansion de la religion chrétienne.

\section{La tahitianisation par la religion}

Les premiers missionnaires protestants venus d'Europe, qui appartenaient à la "London Missio- nary Society", choisirent les Îles Du Vent comme point de départ pour leur travail d'évangélisation. Leur rapide collusion avec le roi Pomare II, qu'ils avaient réussi à convertir, fit de son dialecte tahitien la langue du royaume (Îles du Vent, quelques îles des Tuamotu de l'ouest, plus Tupua'i, Ra'ivavae aux Australes) en même temps que celle de la religion nouvelle (le protestantisme réformé).

La rédaction en tahitien d'un code de lois en 1819 , la traduction de la Bible en 1836, d'un dictionnaire par Davies en 1851, assurèrent au tahitien un réel ascendant sur tous les autres parlers : 
c'était alors la seule langue écrite, et sacrée, de tout le territoire.

En ce début du XIX siècle, l'ensemble du royaume de Pomare se convertit au protestantisme. La Bible en tahitien devint lecture quotidienne aussi bien dans les régions déjà tahitianophones, que dans celles qui pratiquaient une autre langue polynésienne. La première source de tahitianisation fut donc l'évangélisation. En 1819, le roi Pomare II se rendit à l'île de Ra'ivavae, aux îles Australes, qu'il venait juste d'incorporer à son royaume. Avec lui, deux missionnaires tahitiens étaient du voyage, qui restèrent sur place pour évangéliser la population. À la fois langue du pouvoir royal et des serviteurs de Dieu, le tahitien ne vit nulle part sa suprématie contestée. Progressivement, les dialectes locaux furent affaiblis voire supplantés, comme notamment à Ra'ivavae, Tupua'i, ou Rapa.

Le protectorat, instauré en 1842 par la France et le royaume tahitien, ne changea pas l'ordre des choses : le tahitien demeurait la langue principale de tout l'archipel.

Le rôle de la religion dans l'uniformisation linguistique du territoire allait se poursuivre tout au long du Xxe siècle. D’un côté, les îles devenues catholiques - par exemple, Pukarua, Fangatau, Napu$\mathrm{ka}$ - conduisaient leurs offices en latin (du moins jusqu'au concile Vatican II) et, dans une moindre mesure, dans les langues locales. À l'inverse, l'église protestante continua longtemps de conduire ses offices en langue tahitienne. Encore aujourd'hui, dans l'île d'Oparo (Rapa Iti, dans les Australes), les enfants assistent à l'école protestante "du dimanche" en tahitien, et parfois suivent leurs parents aux "tuāro'i", discussions autour de la Bible dans sa traduction tahitienne. Très tôt donc, ces enfants nés dans une des îles les plus éloignées de Tahiti, sont poussés à en maîtriser la langue.

\section{La tahitianisation par l'économie}

Les explorateurs du XVIII ${ }^{\mathrm{e}}$ siècle que furent Wallis, Cook, Bougainville, ne firent que de brefs séjours dans les îles. Si leur présence eut des effets sur les langues du territoire, ce ne fut pas tant par leur usage de l'anglais - fort superficiel - que du fait des inévitables virus apportés par les marins étrangers, et contre lesquels les populations locales n'étaient pas immunisées; dans certaines populations à effectifs réduits, les maladies introduites ont probablement dû fragiliser, indirectement, la transmission de certains parlers du territoire. Mais c'est surtout l'arrivée progressive, au cours de la première moitié du XIXe siècle, des bateaux des chasseurs de baleines puis des santaliers, qui allait marquer le début des influences linguistiques extérieures.

Originaires de divers atolls et îles, les équipages recrutés sur ces bateaux tentaient d'imiter la langue des officiers du bord - en général l'anglais. Ce processus donna d'abord naissance à un sabir maritime assez rudimentaire, à base lexicale anglaise (Tryon \& Charpentier 2004). L'absence de sédentarité, et les changements incessants dans la composition des équipages, interdisaient toute évolution de ces pré-pidgins vers une plus grande stabilité, qui aurait assuré leur survie. Baleines et santal étant devenus rarissimes après 1850, ces éphémères langues de contact disparurent avec les activités qui les avaient fait naître. Ainsi, ce ne sont pas ces premiers parlers maritimes qui allaient bouleverser les équilibres linguistiques ancestraux ; pour cela, il fallut attendre des activités sédentaires, et donc prolongées dans le temps.

Constatant l'absence de mouillage sûr dans la plupart des îles de leur colonie des Marquises, les Français commencèrent à s'intéresser à l'île de Tahiti, cette "Nouvelle Cythère". C'est là qu'ils imposèrent leur protectorat en 1842 . Ils gardèrent pour centre administratif la cité de Papeete, qui avait été choisie comme capitale par la reine Pomare IV en 1827. Profitant de l'excellente rade, ils développèrent un port qui devint centre de tout négoce, de tout échange avec le monde extérieur. Toutes les compagnies de navigation étrangères et françaises, toutes les sociétés faisant du commerce y étaient concentrées. L'ancienne langue de contact des tout débuts du XIX siècle étant devenue depuis longtemps obsolète, le rôle de lingua franca - langue de contact permettant de communiquer d'un archipel à l'autre - revint au tahitien.

Tout déplacement se faisant alors par la seule voie maritime, la langue de communication employée par toutes les populations de l'est ou du sud de la Polynésie devint logiquement celle parlée à Papeete, port d'attache des goëlettes inter-îles, et lieu des périodes de relâche entre deux voyages. En outre, les équipages des bateaux, majoritairement composés de Tahitiens, imposaient aux membres issus des autres archipels l'utilisation de leur langue.

Langue des échanges économiques, le tahitien fut aussi durant plus d'un siècle (1850-1950) la langue des lieux de production des deux seules matières premières du pays : la nacre et le phosphate. 
L'industrie de la nacre (huîtres plates), concentrée dans certaines régions de la Polynésie - Tuamotu du Nord, Mangareva, etc. - requérait de nombreux plongeurs pour récolter les "nacres", ainsi que des travailleurs pour les nettoyer et les vider, seules les coquilles étant commercialisées. Tous ces travailleurs provenaient des différents archipels et parlaient leurs propres dialectes. La langue de contact pour tous était le tahitien - y compris pour les commerçants chinois associés à cette industrie. Encore de nos jours, succédant à l'exploitation de la nacre dans les archipels des Tuamotu et des Gambier, l'industrie perlière, avec ses fermes perlicoles, nécessite une nombreuse main-d'oeuvre introuvable sur place, d'où de nombreux brassages de populations. Ces situations concourent grandement à l'expansion d'une lingua franca pour relier tous les participants au développement: partout il s'agit du tahitien, ou parfois d'une forme légèrement pidginisée mêlant tahitien et français.

L'expansion du tahitien résulta également d'une autre industrie, développée entre 1911 et 1966 : l'exploitation des phosphates de Makatea, île isolée de l'archipel des Tuamotu. De toute la Polynésie française, pour des périodes plus ou moins longues, des migrants vinrent travailler pour la Compagnie Française des Phosphates d'Océanie. Durant leur séjour, leur langue commune était le tahitien, que souvent les migrants réintroduisaient dans leur propre village lors de leur retour. En parallèle, l'implantation du C.E.P., dont nous parlerons plus loin, eut non seulement l'effet de renforcer la "francisation" du territoire (p.57), mais également, d'une manière inévitable, d'accentuer sa tahitianisation, au détriment des autres parlers.

Un corollaire essentiel de ces mouvements de populations pour raison économique fut l'augmentation du nombre d'unions mixtes, associant hommes et femmes originaires de régions différentes de l'archipel. Lorsque l'un des deux parents parle tahitien, c'est généralement sa langue qui va prévaloir dans le couple; si les deux parlent des dialectes différents autres que le tahitien - par exemple marquisien et pa'umotu - aucune de ces deux langues ne s'imposera, et il arrive souvent que le couple finisse par adopter, au sein de la famille, une langue neutre : généralement le tahitien, et parfois, de plus en plus, le français. Le lieu de résidence du couple peut jouer un rôle dans ces choix. Cependant, comme partout ailleurs dans le monde, une famille aura toujours tendance à privilégier la langue jugée la plus "utile" d'un point de vue social ou économique, au sens où on espère qu'elle favorise l'avenir des enfants. C'est ainsi que le tahitien est souvent adoptée dans des familles, y compris lorsque cette langue est étrangère aux deux parents. Depuis deux générations, ce rôle est de plus en plus dévolu au français.

\section{La tahitianisation par le système éducatif}

Depuis une cinquantaine d'années, les institutions en place dans le pays, en particulier l'école, se sont donné pour mission d'étendre l'usage de la langue française à travers le territoire. ${ }^{23}$ Pourtant, en même temps qu'il renforce la position du français, ce système éducatif a également pour effet collatéral, paradoxalement, le renforcement de la langue tahitienne au détriment des autres vernaculaires de l'archipel.

\section{La tahitianisation scolaire indirecte}

Jusqu'au milieu du XIX ${ }^{e}$ siècle, l'éducation avait été laissée aux soins des Églises, souvent dépourvues de moyens matériels et humains pour remplir cette mission. Il en a résulté jusqu'au milieu des années 1960 des situations sociolinguistiques très différentes d'un archipel à l'autre, voire d'une île à l'autre; ici le patrimoine culturel était assez bien conservé, là il était sur la voie d'une complète disparition. Ce n'est qu'après la Seconde Guerre Mondiale, à la suite du choix des populations polynésiennes de devenir Territoire d'Outre-Mer (TOM), que l'État français commença à investir dans l'éducation: construction d'écoles et de collèges, envoi de coopérants, formation de maîtres locaux. Copié sur le système éducatif français dans lequel l'école est obligatoire jusqu'à 16 ans et l'enseignement gratuit de la maternelle à la fin de tout cycle universitaire, le système scolaire de la Polynésie française est sans équivalent dans le Pacifique nonfrancophone. Obligatoire et gratuit, il permet en théorie de donner une chance à tous.

Cependant, précisément par sa vocation universelle, cet enseignement couvre l'ensemble du territoire. Dans la mesure où il se déroule exclusivement en français - puis, plus tard, en tahitien - un tel système éducatif a pour conséquence évidente une acculturation généralisée, sans aucune considération pour la diversité linguistique de la Polynésie française. Qui plus est, ce système est pyramidal, dans le sens où la réussite de tout élève implique qu'il devra poursuivre ses études, ou trouver un travail, dans l'île principale de Tahiti. Il

23 Ce processus de "francisation" sera évoqué plus loin (p.63). 
résulte de cette situation une expansion de la langue française, qui met en danger les langues et cultures polynésiennes; mais aussi une consolidation du centralisme de Tahiti, qui condamne à terme la survie des langues autres que le tahitien. Ce que les missionnaires n'avaient pas réussi à éradiquer, ce système éducatif pyramidal et assimilationiste a bien failli l'achever en quatre décennies.

Le problème principal de cette situation n'est pas tant la présence - historiquement nécessaire et socialement inévitable - des langues dominantes que sont le français et le tahitien. Avant tout, c'est l'idéologie monolingue qui fait des ravages : à savoir, l'idée qu'un enfant ne peut pas apprendre correctement plusieurs langues, et qu'il lui faut nécessairement en choisir une seule aux dépens des autres. Il est bien connu, pourtant, que le plurilinguisme est une situation parfaitement normale non seulement dans les sociétés traditionnelles du Pacifique, mais aussi également dans la plupart des sociétés du monde. Le monolinguisme, en revanche, est une anomalie historique, en partie influencée par l'histoire de l'Europe, et la notion moderne d'État-nation héritée des Romantiques allemands. Plutôt qu'imposer à la Polynésie française cette même idéologie monolingue, il serait plus profitable de donner les moyens à ses citoyens de parler plusieurs langues durant tout au long de leur vie - aussi bien les langues locales que les langues nationales ou internationales. En somme, la question n'est pas de savoir si le français ou le tahitien devraient être enseignés à l'école - car c'est bien entendu nécessaire ; mais plutôt, si cet apprentissage doit forcément impliquer l'élimination des autres langues du territoire.

Aujourd'hui, tous les enfants du territoire sont soumis au même cursus, du primaire au supérieur. La plupart d'entre eux, en dehors des tahitianophones et dans une moindre mesure des Marquisiens et des habitants de Rurutu/Rimatara dans les Australes, sont coupés très jeunes de leurs propres cultures ancestrales. Les exemples qui suivent suffiront pour illustrer cette acculturation précoce par le biais de l'école.

Des enfants nés dans les atolls de Pukarua, Fangatau, Napuka par exemple ont de fortes chances d'apprendre la langue locale au sein de leur famille, au moins avec la génération de leurs grandsparents. Dans la mesure où leur communauté possède une école maternelle, ils pourront commencer leur scolarisation dans leur environnement familier, en contact avec leur propre langue ; cependant, l'enseignement reçu à l'école sera en français, et le tahitien sera déjà présent à travers les médias. À la fin de l'école primaire, vers onze ans, ils subissent une première rupture avec leur environnement familier : le seul enseignement secondaire dans la région prend la forme d'un internat sur l'atoll de Hao, où ils vont côtoyer des élèves d'autres atolls. Désormais, la langue qu'ils auront apprise dans leur enfance se trouve déjà en minorité dans la vie quotidienne, concurrencée par d'autres langues. En outre, comme beaucoup d'enseignants polynésiens parlent tahitien, c'est souvent la seule langue polynésienne qu'ils vont entendre parlée par des adultes, et éventuellement commencer à parler eux-mêmes - en concurrence avec le français. Pour les meilleurs élèves, la poursuite d'études plus avancées impliquera une seconde rupture: ils devront quitter leur archipel des Tuamotu pour se rendre à la capitale Papeete. Là, l'exposition au tahitien deviendra quotidienne : à Tahiti, on finit toujours par adopter soit le tahitien comme langue principale, soit le français. Arrivés à l'âge adulte, les enfants n'auront finalement vécu de manière continue dans leurs atolls que quelques années - pas assez pour en maîtriser tout à fait la langue et la culture. C'est ainsi que la scolarisation, en dépit de tous ses bienfaits, résultera typiquement dans l'uniformisation de tout le territoire, et la perte de sa diversité linguistique.

Aux îles Marquises, l'influence du tahitien par le biais de l'école se fait plus tardivement, dans la mesure où l'archipel possède écoles primaires et collèges. Un enfant de l'île Fatu Hiva par exemple parlera le dialecte marquisien de ses ancêtres durant toute la durée de sa scolarité primaire puis à onze ans au collège à Atuona (Hiva $\mathrm{Oa}$ ), il se familiarisera avec les différences entre le dialecte local et le sien. Parce que les divisions dialectales n'entravent pas une intercompréhension généralisée, il ne cessera donc pas de parler marquisien. Le tahitien restera pour lui une langue étrangère, celle des médias, ou de certains Tahitiens de passage, fonctionnaires ou touristes. C'est seulement au collège que les enfants marquisiens commencent à être réellement confrontés à la langue de la capitale. Ils doivent consacrer plusieurs heures hebdomadaires à son apprentissage, avec pour prétexte de les préparer à un éventuel départ dans un lycée à Tahiti.

Comme les petits Marquisiens, les enfants nés dans les îles de Rurutu et Rimatara dans l'archipel des Australes bénéficient d'un collège dans leur propre aire linguistique : ceci permet de retarder et limiter la tahitianisation précoce, qui fait des ravages ailleurs dans le pays. 
Ce système éducatif pyramidal avec, pour fin d'études réussies, trois voire quatre années à Tahiti, a pour but de faire émerger une élite de type occidental. En ne prenant pas en compte les cultures et les langues locales, ce système contribue de fait à leur disparition.

Nous proposons de décrire ce processus historique en termes de "tahitianisation scolaire indirecte". En effet, le paradoxe est que ce système éducatif, tel qu'il est organisé, résulte en l'expansion de la langue tahitienne à travers l'archipel, alors même que la langue principale d'enseignement n'est pas le tahitien, mais le français. Alors que l'école est une cause directe d'expansion du français - nous le verrons plus loin - elle est donc aussi une cause indirecte d'expansion du tahitien.

Longtemps, le tahitien ne s'est diffusé à travers l'école que de cette manière que je viens d'évoquer - parce qu'elle est la lingua franca employée entre élèves d'îles différentes, ou entre professeurs et élèves. Mais comme nous allons le voir maintenant, l'école contribue depuis peu à l'expansion du tahitien à travers les archipels de manière plus directe: en en faisant une langue d'enseignement. S'il est vrai que ce dernier mouvement est historiquement une victoire pour la consolidation de la langue tahitienne face à la pression du français, en revanche il a pour effet d'augmenter davantage encore la pression du tahitien sur les autres langues polynésiennes du pays. Ceci s'explique beaucoup par les ambiguïtés de la loi Deixonne.

\section{Les ambiguïtés de la loi Deixonne}

L'État français est connu pour son modèle "jacobin" de centralisation politique et culturelle, y compris du point de vue linguistique. En 1951, une première brèche dans cette uniformisation linguistique fut apportée par la loi Deixonne, qui autorisa l'enseignement des langues régionales de France. Cependant, cette loi présentait tant d'ambiguïtés et d'imperfections, qu'elle ne fit rien pour vraiment préserver la diversité linguistique de la Polynésie française.

Tout d'abord, la version initiale de cette loi Deixonne était restrictive, puisqu'elle ne reconnut au départ que quatre langues régionales de France - le breton, le basque, le catalan et l'occitan. Il fallut attendre 1981 pour que fût introduite la langue tahitienne (extension de la loi Deixonne par décret $n^{\circ} 81-553$ du 12 mai 1981).

S'il fut une bonne nouvelle pour les défenseurs de la langue tahitienne, le décret avait le défaut d'ignorer totalement les autres langues de Polynésie française - marquisien, mangarévien, pa'u- motu... Par ailleurs, l'article 1er de ce décret fait preuve d'une certaine ambiguïté :

Les articles (...) de la loi du 11 janvier 1951 (...) relatifs à l'enseignement des langues et dialectes locaux sont applicables dans la zone d'influence du tahitien.

Que fallait-il donc comprendre comme "la zone d'influence du tahitien" ? Si l'on prend le tahitien en tant que lingua franca, son "aire d'influence" recouvre l'ensemble du territoire - ce qui revient à nier la diversité linguistique de la Polynésie française. $\mathrm{Si}$, au contraire, on considère le tahitien comme une langue vernaculaire parmi d'autres, alors son enseignement devrait se limiter à la zone où cette langue est l'unique vernaculaire local : les îles du Vent, les îles Sous-le-Vent, le nord-ouest des Tuamotus, et Tupua'i (cf. Map 1 p.21).

Devenue autonome en 1984, la Polynésie française hérita de ces ambiguïtés. Et bien souvent, la seule langue enseignée dans les écoles à côté du français allait être la langue de Tahiti. Ainsi, le premier statut d'autonomie de la Polynésie française stipulait : ${ }^{24}$

"La langue tahitienne est une matière enseignée dans le cadre de l'horaire normal des écoles maternelle et primaire. Cet enseignement est organisé comme matière facultative et à option dans le second degré. Sur décision de l'assemblée territoriale, la langue tahitienne peut être remplacée dans certaines écoles maternelles et primaires par l'une des autres langues polynésiennes."

La formulation même de ce texte législatif révèle sans ambiguïté l'impérialisme tahitianophone qui régnait alors. En effet, le choix du tahitien s'y imposait par défaut à tout le territoire, tandis qu'il fallait une dérogation spéciale, accordée par l'assemblée de la capitale, pour enseigner "l'une des autres langues polynésiennes".

Par ailleurs, la portée de la loi Deixonne était largement amoindrie par le caractère facultatif ajouté à différents articles : non seulement cet enseignement serait facultatif pour les élèves, mais l'existence même d'un tel enseignement dépendait $\mathrm{du}$ bon vouloir de l'instituteur lui-même. Ainsi, c'est sur sa demande que l'instituteur est autorisé à faire chaque semaine une heure d'activité dirigée portant sur la lecture, l'écriture des langues locales. De même, l'article 5 de la loi propose l'organisation facultative de cours et stages dans

24 Loi n84-820 du 6 septembre 1984 portant statut du territoire de la Polynésie française, art. 90. Nous remercions Jacques Vernaudon d'avoir attiré notre attention sur ce texte de loi. 
les écoles normales, portant sur la langue, le folklore, la littérature locale. L'épreuve de langue au baccalauréat est également facultative.

Enfin, une autre ambiguïté résultait non pas de la loi Deixonne elle-même, mais de la notion de reo $\boldsymbol{m a} \bar{a}^{\prime} o h i$ qui lui fut associée dans le discours officiel. En tahitien, reo $m \bar{a}^{\prime}$ ohi signifie 'langue autochtone'. De la même façon, une plante, un oiseau etc. qualifiés de $\boldsymbol{m} \bar{a}^{\prime}$ ohi sont "endémiques", "indigènes". En principe, cette expression reo $\boldsymbol{m} \bar{a}^{\prime}$ ohi devrait donc pouvoir désigner n'importe quelle langue autochtone du territoire, qu'il s'agisse du tahitien (reo Tahiti), du marquisien ou du reao... Pourtant, l'équation reo $\boldsymbol{m} \overline{\boldsymbol{a}}^{\mathbf{c}} \mathbf{o h} \mathbf{i}=$ tahitien finit par s'imposer dans les esprits, ainsi que dans le discours officiel des années 1980-1990, et ce jusqu'à très récemment.

Durant les deux décennies qui suivirent le Décret de 1981, c'est donc la langue de Tahiti et elle seule qui bénéficia de cette reconnaissance dans le système éducatif. Seule langue polynésienne reconnue par l'État, c'était aussi, de fait, la seule langue polynésienne enseignée. Le tahitien commença à être enseigné non seulement à l'Université, où une chaire de Langues et Civilisations polynésiennes fut ouverte, mais aussi, conformément au cadre de la Loi Deixonne, dans les collèges et lycées.

D'un côté, on peut regretter à juste titre que le tahitien ne bénéficie encore, dans le système scolaire, que de quelques heures par semaine à peine - si peu par rapport à la place de la langue française encore omniprésente. Mais de l'autre côté, même ce faible nombre d'heures constitue une occasion supplémentaire permettant à la langue tahitienne de s'imposer toujours un peu plus dans les aires linguistiques marquisienne et pa'umotu. Dès le plus jeune âge, les enfants de tout le territoire apprennent que la seule langue polynésienne de culture, la seule dotée d'une écriture et d'un prestige, est celle de Tahiti.

Quant aux autres langues du territoire, leur enseignement n'était pas envisagé par l'État central. Il ne fut mis en place que de manière ponctuelle et informelle, là où des enseignants motivés se jugaient aptes à remplir une telle tâche - ex. à Mangareva, aux Marquises, à Rimatara. À l'inverse, les enseignants en poste aux Tuamotu étant presque toujours eux-mêmes originaires de la zone tahitianophone, ce sont des rudiments de tahitien qu'ils enseignent à leurs élèves, quelle que soit la langue maternelle de ces derniers. Finalement, il aura fallu un changement de gouvernement pour que les langues vernaculaires minoritaires fussent intégrées au système d'enseignement général.

\section{Histoire politique récente : Centralisation linguistique et prises de conscience}

Le nom de Tahiti est trop souvent utilisé, par métonymie, pour désigner le pays dans son ensemble. Cette pratique est davantage qu'une facilité de langage : c'est une véritable idéologie, inspirée en partie du modèle centralisateur français. Le jacobinisme que je viens d'illustrer du point de vue linguistique, n'est en réalité qu'une des facettes d'un phénomène plus général, communément décrit comme l'impérialisme de la part de Tahiti.

Durant les deux décennies qui ont suivi l'autonomie de la Polynésie française, les hommes politiques continuèrent ce centralisme obsessionnel autour de Tahiti, y compris lorsqu'ils étaient eux-mêmes originaires d'autres points de l'archipel - tel le premier d'entre eux, Gaston Flosse, natif de Mangareva. Tout semblait s'organiser autour d'un modèle simple : un pays, une île-capitale, une langue.

Ainsi, le terme de "Polynésie française", instauré en 1957, fut considéré à juste titre comme un rappel de l'ancienne dépendance coloniale. Au milieu des années 1990, le désir de trouver un autre nom pour le territoire, donna lieu à un nouveau terme officieux: "Tahiti Nui". Littéralement "le Grand Tahiti", ce terme implique que l'ensemble immense de la Polynésie française, avec ses cinq archipels, peut légitimement être réduit au statut de simples dépendances de l'île de Tahiti. D’ailleurs, la traduction souvent donnée de ce terme "Tahiti Nui", y compris par le très officiel Ministère du Tourisme, n'est-elle pas Tahiti et ses îles? Au lieu d'être présentée pour ce qu'elle est - une île parmi tant d'autres - Tahiti devient une entité abstraite, centre de tout, synthèse de tout le pays.

C'est ainsi que, durant la décennie 1990, tous les navires administratifs furent appelés "Tahiti Nui", et que la compagnie aérienne longs-courriers devint "Air Tahiti Nui". Parallèlement, contre l'avis même des habitants, on incita toutes les femmes enceintes des Tuamotu ou des Gambier à venir accoucher à Tahiti, avançant des raisons sanitaires. Les registres d'état-civil dans ces îles n'enregistrent plus que des décès et toutes ces mères des îles et atolls en question donnent naissance à des bébés "tahitiens" - du moins enregistrés comme tels.

Cet extrême centralisme, faisant de Tahiti le centre névralgique du pays, eut bien sûr des conséquences sur le plan linguistique. Le tahitien est devenu la seule langue polynésienne utilisée dans 
les médias ${ }^{25}$, la seule parlée à l'assemblée de Polynésie (avec le français), la seule écrite dans certains ministères comme celui de la santé, de l'agriculture, etc., la seule enseignée enfin dans les collèges. Au niveau administratif, une diglossie avait été mise en place avec le français comme langue dominante parce qu'officielle, et le tahitien pour unique langue seconde.

Dans les autres archipels, cette omniprésence du tahitien fut parfois mal acceptée, et fut la cause de ressentiment. Refusant d'être des citoyens d'un "grand Tahiti", les Marquisiens préféraient donner au bateau assurant les liaisons entre leurs îles le nom de "Ka'oha Nui" (de ka'oha 'bonjour, bienvenue' en marquisien). Les Pa'umotu, très nombreux à Papeete la capitale, se hasardaient rarement à parler leur langue. Ils étaient trop souvent affublés du dépréciatif "kaina" (gens mal dégrossis). De même, les Reao étaient stigmatisés comme "mangeurs de savates", les gens de Napuka comme "napua'a" ou "napuaka" (les cochons). Le plus souvent ignorés, souvent infériorisés, rares étaient les natifs des îles à songer à défendre leur patrimoine culturel et transmettre leur langue: on se résignait à l'apprentissage du tahitien.

La mise en place d'une politique plus ouverte en matière de langues vit le jour en 1998, lorsque Mme Louise Peltzer - professeur de langues polynésiennes à l'Université de la Polynésie française fut nommée Ministre de la Culture (1998-2004). Ayant besoin des voix des élus marquisiens, le gouvernement de Gaston Flosse prit alors en compte les revendications de ces derniers en matière de langues et de culture, infléchissant ainsi sa précédente politique du tout tahitien. Une académie fut mise en place aux îles Marquises; une journée des langues fut instaurée; un prix littéraire du Président fut attribué pour une œuvre écrite en langue autochtone ; beaucoup d'associations culturelles, et pas seulement tahitiennes, furent subventionnées. Ces décisions marquèrent la reconnaissance de facto du plurilinguisme du territoire. Le progrès était réel, mais néanmoins partiel : il en faudrait davantage pour entamer la suprématie du français ou du tahitien.

En 2004, un changement de majorité politique inattendu amena au pouvoir une nouvelle coalition - incluant le parti indépendantiste Tãvini Hui-

25 D’abord de nombreuses radios dans les années 1970, arrivée de la télévision entre 1980 - 1990, puis enfin pose des premières paraboles de télévision par satellite entre 1990 et 2000. ra'atira - avec un mot d'ordre : taui roa (en tahitien: changer complètement). Dans le domaine linguistique, le changement fut réel, mais long à se mettre en place. La grande majorité des dirigeants indépendantistes est originaire de Tahiti, où se situe leur lieu de résidence. Pour eux, le tahitien était depuis toujours la langue de la revendication, langue dans laquelle on s'adressait aux seuls ta'ata tumu ("gens du pays"), par contraste avec le français. Pour ces hommes politiques, parler tahitien en tout lieu, en toute circonstance revient à affirmer son identité "nationale", à refuser toute allégeance à l'égard de l'État français, voire à exclure les Popa'ā (Européens vivant dans le pays, qui ne parlent aucune langue polynésienne). Au bout du compte, si les relations entre français et tahitien furent redéfinies lors de cette période, en revanche cela n'impliqua aucun progrès pour le maintien des vernaculaires locaux - bien au contraire. La politique centralisatrice fut poursuivie et même renforcée, et les autres langues exclues de la vie politique. Après 2004, tout ministre en déplacement - y compris le Président du pays - continuait à ne s'adresser aux citoyens qu'en tahitien, où qu'il soit. Il était pour le moins paradoxal de constater que les pourfendeurs même de "l'impérialisme linguistique" du français, finissaient par perpétuer une forme d'“impérialisme" du tahitien.

Si cette période politique eut des effets positifs en matière de diversité linguistique, ce ne fut pas tant l'effet de l'action du gouvernement que le résultat d'une prise de conscience sur le terrain. À force de réclamer haut et fort le changement par rapport à des années de pouvoir autocrate, cette période ouvrit la voie à toute forme de contestation, et redonna confiance aux diverses minorités culturelles et linguistiques. Ainsi, lorsque la nouvelle coalition au pouvoir - sous la houlette de Jacqui Drollet, alors vice-premier ministre - décida de parler exclusivement tahitien au sein de l'assemblée de Polynésie française, un élu marquisien, René Kohumoetini, décida à son tour de ne parler que sa propre langue: il dénonçait ainsi l'attitude autoritaire de la majorité en place, en révélant l'absurdité d'imposer une seule langue au sein de l'assemblée d'un pays multilingue. D'autres représentants du peuple, qui souvent avaient recours au tahitien, n'utilisèrent plus que le français, pour protester contre le nouveau centralisme. La libération des esprits qui suivit le changement de majorité, encouragea les Pa'umotu de la capitale jusqu'alors qualifiés de "kaina" (arriérés) - à parler leur propre langue entre eux en public, et à affirmer leur identité, par exemple, à travers des fes- 
tivals qui font aujourd'hui leur fierté. La plupart des chanteurs de renom du pays ne sont-ils pas originaires de leur archipel ? Cette affirmation des cultures insulaires se retrouve notamment au cours du festival annuel des arts, le "Heiva". A Tahiti, en 2004, le groupe culturel de Rapa Iti remportait plusieurs premiers prix, souvent en ne s'exprimant que dans sa langue, le "reo Oparo".

Aux Marquises, le renouveau culturel associé à la recherche d'une identité propre ont conduit les enseignants du primaire à intégrer langues et cultures locales dans le cursus scolaire. Aujourd'hui, des compétitions (art oratoire, mimiques, maîtrise de la langue...), sont organisées entre classes de même niveau d'une même île, et bientôt entre les cinq îles de l'archipel.

La classe politique tahitienne, très occidentalisée dans son mode de vie, n'était pas sensibilisée à de telles questions. Ainsi, y compris au cours de cette ère du Taui (du "changement"), on constata peu d'innovations majeures en matière de préservation linguistique - à une exception près. Le nouveau Ministre de l'Éducation, Jean Marius Raapoto, était issu d'une famille protestante au sein de laquelle l'amour de la langue (le tahitien) était élevé en culte. Ayant terminé ses études supérieures par une thèse en linguistique, il avait ensuite fait carrière comme enseignant: cette formation lui fit comprendre que la défense des langues et des cultures passait par leur enseignement. Une telle approche, qui irait de soi en Europe, apparaissait comme une révolution en Polynésie française. Révélateur de cette nouvelle attitude vis-à-vis du plurilinguisme dans le territoire, était le nom complet choisir pour ce ministère: "Ministère de l'Éducation, de l'Enseignement supérieur et de la recherche, chargé du plurilinguisme et de la promotion des langues polynésiennes".

C'est à cette occasion que fut levée l'ambiguïté de l'expression reo $\boldsymbol{m} \bar{a}^{\prime}$ ohi "la langue autochtone", mentionnée plus haut. L'expression fut alors actualisée pour désigner précisément l'ensemble des dialectes de la Polynésie française (reo Tahiti "tahitien", 'eo 'Enata/'Enana "marquisien", marau Reao "le reao", reko Takapoto "le vahitu de Takapoto", tako Fangatau "le fangatau", geo Ra'ivavae "le ra'ivavae"...). En bon linguiste, le ministre s'en tint au sens exact de l'expression et rompit avec l'habitude prise de limiter l'enseignement du reo $\boldsymbol{m} \bar{a}^{\prime}$ ohi au seul enseignement du tahitien : ce geste équivalait, pour la première fois, à une reconnaissance officielle du plurilinguisme du pays.

Profitant de l'ambiguité de la loi Deixonne et du décret qui s'ensuivit, il fut ainsi décidé de privilégier l'enseignement des dialectes locaux constituants du reo $\boldsymbol{m} \bar{a}^{\prime} \mathbf{o h i}$, partout où ils étaient encore en usage. Pour ce faire, des stages spécifiques de formation furent organisés, et un certain nombre d'enseignants déjà formés furent invités à s'initier à l'enseignement de leurs propres langues maternelles : celles-ci étaient soudain reconnues comme langues $\boldsymbol{m} \bar{a}^{\prime}$ ohi les unes comme les autres. Les critiques de la part des tenants du monolinguisme tahitien restèrent modérées, et après un nouveau changement de gouvernement, cette "expérience" - appellation sous laquelle cette politique nouvelle avait été prudemment présentée - fut poursuivie, tant aux Marquises qu'aux Tuamotu ou aux Australes.

\section{Tendances et pesanteurs de la situation sociolinguistique}

L'hégémonie du tahitien n'a fait que se renforcer au cours des dernières années (depuis 2008), mettant encore plus en péril l'existence même des autres langues.

Les différents gouvernements locaux ont fait le choix délibéré de promouvoir le seul reo tahiti. En outre, la crise économique qui touche la perliculture obligea les travailleurs sous contrat à rester dans les atolls où ils travaillaient plutôt que de rentrer chez eux, ce qui renforce l'usage d'un tahitien limité. Bien peu regagnent leurs îles natales mais s'entassent dans la capitale Papeete, où ils se paupérisent. Ces immigrés, locuteurs de dialectes étrangers aux îles de la Société, sont obligés de faire usage du reo tahiti, langue dominante qu'ils maîtrisent plus ou moins.

Confrontés à des variations langagières multipes, les jeunes se tournent souvent vers le français, qui devient alors la "première langue" d'une majorité d'entre eux. 


\section{La francisation à marche forcée}

Malheureusement, le taui ("changement") arrive un peu tard. Depuis une cinquantaine d'années, les choix politiques et stratégiques au niveau de l'État avaient donné à la langue française un rôle prépondérant dans de nombreux domaines de la vie du pays.

La décision du gouvernement du général de Gaulle en 1963 de transférer le Centre d'Expérimentation nucléaire du Pacifique (C.E.P.), de Reggane en Algérie vers la Polynésie française, allait avoir des conséquences culturelles et linguistiques encore plus importantes que les deux siècles de contact entre Polynésiens et Européens.

Toute la population de la Polynésie française fut plus ou moins impliquée par cette décision. Dès lors, la suprématie du seul tahitien en tant que lingua franca avait vécu: le français, en moins d'une décennie, allait devenir la langue dominante. Les facteurs économiques qui avaient conduit à des changements linguistiques - en particulier une tahitianisation lente du pays - par des concentrations et migrations de populations, se retrouvèrent soudain décuplés au profit, cette fois, de la langue du colonisateur venu d'Europe.

Pour construire les innombrables infrastructures nécessaires à ces essais nucléaires (routes, pistes d'atterrissage, abris, logements...), il fut fait appel aux populations locales. Les autorités militaires envoyèrent des agents recruteurs dans tous les archipels : au cours des années 1960, pas moins de 13000 Polynésiens furent employées par le C.E.P. Les mouvements de population continuèrent de cette manière durant une trentaine d'années, jusqu'au démantèlement définitif du Centre en 1995. Une très large portion de la population masculine en âge de travailler participa donc pour des durées plus ou moins longues à ce programme. Absents de leurs communautés, ces hommes apprirent à vivre d'un salaire, et délaissèrent les activités traditionnelles qu'étaient la pêche et l'agriculture. Le riche vocabulaire appris des anciens devenait désormais inutile, dans cette société nouvelle reposant sur l'échange monétaire et l'usage du français.

L'émigration vers Papeete se fit massive. On y trouvait des emplois, et plus ou moins du logement; c'est ainsi que des milliers de familles quittèrent leur communauté, et vinrent s'entasser à la périphérie de la capitale. Les conséquences sociales, culturelles et linguistiques de ce pro- cessus allaient s'avérer immenses pour le pays : aucun lieu de la Polynésie française n'allait échapper à ce processus massif d'exode rural. Îles et atolls autres que Tahiti perdaient une large partie de la génération la plus dynamique - celle qui a des enfants - supprimant ainsi un maillon essentiel dans la transmission du savoir ancestral.

À Papeete, tous ces immigrants étaient dans l'incapacité de parler leur langue, si ce n'est dans l'étroit cadre familial. En dehors de la maison, le tahitien devenait lingua franca, langue seconde d'une large partie de la population polynésienne.

Par ailleurs, en quête d'un emploi, tous les nouveaux venus se trouvaient contraints dans leur activité professionnelle de parler français. En effet, l'installation et le fonctionnement du centre d'essais nucléaires impliquaient un afflux massif de populations d'origine métropolitaine. Ces nouveaux venus avaient un profil très différent des traditionnels "Petits Blancs" qui arrivaient sur les bateaux des Messageries maritimes, avec pour rêve de vivre "à la tahitienne" : pendant des générations, ces voyageurs et petits colons avaient montré qu'ils étaient disposés à se fondre dans la population, voire à faire l'effort d'apprendre la langue locale, ne fût-ce qu'un minimum. Rien de tels avec les nouveaux venus du C.E.P.: qu'ils soient militaires, fonctionnaires ou autres, ces nouveaux "Farāni" (Français) venaient en Polynésie pour de courts séjours - quelques années au plus; leur venue n'avait rien à voir avec un quelconque amour du pays ou de ses habitants, encore moins de ses cultures ou de ses langues. Ces métropolitains étaient nombreux, et pourvus d'un fort pouvoir d'achat - assez pour imposer en tout lieu leur mode de vie et leur langue.

Lorsque des Polynésiens se retrouvaient entre eux, ils pouvaient bien sûr parler la lingua franca tahitienne; cependant l'omniprésence des "Po$\boldsymbol{p} \boldsymbol{a}^{\prime} \overline{\boldsymbol{a}}^{\prime \prime}$ (Européens) rendait ces circonstances assez rares, et l'usage du français devenait quasi obligatoire. Progressivement, on finissait par parler français en toutes circonstances à Papeete : insidieusement, la langue venue d'Europe avait pris le pas sur le tahitien dans la capitale. Le sentiment amer des Polynésiens est ainsi exprimé par le poète tahitien Henri Hiro ${ }^{26}$ (1944-1990) :

${ }^{26}$ Henri Hiro - cité par Bruno Saura (2004). 
“Si tu étais venu chez nous, nous t'aurions accueilli à bras ouverts.

Mais tu es venu ici chez toi, et on ne sait pas comment t'accueillir chez toi".

Ce processus de "francisation" s'étendit sur trois décennies, et quasiment deux générations de Polynésiens en furent les acteurs. Cependant, l'impact de cette acculturation fut inégal selon les lieux.

Les essais nucléaires à proprement parler se situaient au sud de l'archipel des Tuamotu. Autour de la zone d'exclusion comprenant les atolls de Moruroa et Fangataufa furent implantées, dans les îles et atolls alentour, différentes bases d'observation où séjournait du personnel militaire. Que ce soit à Reao, Pukarua, Tureia, Mangareva, Rapa Iti, la proportion de militaires par rapport aux populations locales entraîna de profonds bouleversements dans les sociétés traditionnelles, pour qui le français n'avait été jusqu'alors qu'une langue d'enseignement et rien de plus. La recrudescence de mariages mixtes, la naissance de nombreux "demis" métis, conduisirent à un abandon des cultures et langues locales au seul profit du français. À la fin du centre d'essais nucléaires, ces îles virent partir la plupart des couples mixtes, ainsi que les nombreux jeunes métis nés de ces unions.

Sur des bases arrières tel que l'atoll de Hao, le mélange de populations impliquait, comme à Ta- hiti, un usage occasionnel du tahitien, mais plus souvent du français. Des travailleurs de toutes les communautés de Polynésie avaient séjourné à Moruroa ou Hao, lieux où l'usage du français était incontournable au moins avec les nombreux militaires. En trente ans, le français s'était généralisé.

Comme les séjours des métropolitains étaient souvent relativement courts, et que les départs et retours des travailleurs étaient échelonnés, on aurait pu penser que l'usage du français, langue moins adaptée à la vie des îles, allait se déliter. Mais c'eût été sans compter l'apport de l'école. Après la scolarisation de trois générations en langue française, cette langue était généralement comprise par les moins de soixante ans. Le français devint donc lingua franca pour tous, quelle que soit leur connaissance des langues traditionnelles. Aujourd'hui, comme on l'a vu (p.18), le français est devenu la première langue familiale pour près de $70 \%$ de la population.

Cette francisation généralisée était en partie un objectif déclaré des institutions, et parfois la simple conséquence indirecte d'événements en apparence fortuits. L'accélération de ce phénomène au cours des dernières décennies conduit à s'interroger sur la place future des langues polynésiennes dans le pays. Dans certains cas, se pose même le problème de leur survie. 